\title{
Diseño de un software para la generación de certificados de calibración en metrología térmica
}

\author{
Design of software for the generation of calibration certificates in thermal metrology
}

\author{
F. J. Moreno-Angulo ; D. E. Ochoa-Tuirán iD ; C. A. Pedraza-Yepes ; ; J. E. González-Coneo ; J. \\ D. Hernández-Vásquez
}

\begin{abstract}
In the industrial sector, metrology allows to support the quality of the processes, because, in all these, physical quantities intervene that influence each stage of these and whose effects are reflected in the quality of the final product. These quantities must be measured to take control actions and ensure that their effect will not be negative on the requirements of the products. Measuring instruments, as well as the metrological reliability of their calibration, based on the use of software and computerized systems, become major challenges for industrial metrology. In that order of ideas, the main objective of this work is to explain the methodology for the design of software that allows generating calibration certificates in thermal metrology. The work was motivated due to challenges imposed by Accredited Laboratories to optimize their processes. The methodology adopted, based on the programming of computational algorithms in a C-Sharp language $(\mathrm{C}$ \#) made it possible to: (i) perform a complex uncertainty analysis (GUM); (ii) estimate the adjusted calibration curve from the consecrated theory of ordinary least squares and (iii) validation from real experimental data. The consolidated results allowed us to conclude that the software developed is aligned with the requirements of ISO / IEC 17025: 2017, in addition to being an effective tool for increasing the metrological reliability of industrial processes, thus contributing to the advancement of knowledge in the metrology.
\end{abstract}

Index Terms - calibration certificate; measurement uncertainty; temperature measurement; thermal metrology; software in metrology.

Resumen- En el sector industrial, la metrología permite respaldar la calidad de los procesos, debido a que, en todos estos, intervienen magnitudes físicas que influyen sobre cada etapa de estos y cuyos efectos se ven reflejados en la calidad del producto final. Estas magnitudes deben ser medidas para tomar acciones de control y garantizar que su efecto no será negativo en los requerimientos de los productos. Los instrumentos de medición, así como la confiabilidad metrológica de su calibración, a partir de

Este manuscrito fue enviado el 20 de agosto de 2019 y aceptado el 15 de marzo de 2020

Francisco Javier Moreno Angulo es Ingeniero Mecánico egresado de la universidad del Atlántico. Puerto Colombia, Colombia (e-mail: fjmoreno@ mail.uniatlantico.edu.co).

David Enrique Ochoa Tuirán es Ingeniero Mecánico egresado de la universidad del Atlántico. Puerto Colombia, Colombia (e-mail: dochoa@mail.uniatlantico.edu.co).

Cristian Pedraza-Yepes Profesor de planta de la Universidad del Atlántico pertenece al grupo de investigación CONFORMAT. Puerto Colombia, Colombia (e-mail: cristianpedraza@mail.uniatlantico.edu.co). uso de softwares y sistemas computarizados, se tornan grandes desafíos para la metrología industrial. En ese orden de ideas, el objetivo principal de este trabajo es explicitar la metodología para el diseño de un software que permita generar certificados de calibración en metrología térmica. El trabajo fue motivado debido a desafíos impuestos por Laboratorios Acreditados para optimizar sus procesos. La metodología adoptada, basada en la programación de algoritmos computacionales en un lenguaje $\mathrm{C}$ Sharp (C\#) posibilitó: (i) realizar un complejo análisis de incertidumbre (GUM); (ii) estimar la curva de calibración ajustada a partir de la teoría consagrada de los mínimos cuadrados ordinarios $y$ (iii) validación a partir de datos experimentales realies. Los resultados consolidados permitieron concluir que el software desarrollado se encuentra alineado a las exigencias de la ISO/IEC 17025:2017, además de constituirse una herramienta eficaz para el aumento de la confiabilidad metrológica de los procesos industriales, contribuyendo así, al avance del conocimiento en la metrología.

Palabras claves - certificado de calibración; incertidumbre de medición; medición de temperatura; metrología térmica; software en metrología.

Resumo - No setor industrial, a metrologia permite apoiar a qualidade dos processos, pois, em todos esses, intervêm quantidades físicas que influenciam cada estágio destes e cujos efeitos se refletem na qualidade do produto final. Essas quantidades devem ser medidas para executar ações de controle e garantir que seus efeitos não sejam negativos nos requisitos dos produtos. Os instrumentos de medição, bem como a confiabilidade metrológica de sua calibração, com base no uso de software e sistemas computadorizados, tornam-se grandes desafios para a metrologia industrial. Nessa ordem de idéias, o principal objetivo deste trabalho é explicar a metodologia para o design de um software que permita gerar certificados de calibração em metrologia térmica. $O$ trabalho foi motivado devido aos desafios impostos pelos Laboratórios Credenciados para otimizar seus

Jorge Enrique González-Coneo Profesor de tiempo completo en la Universidad de la Costa (CUC). Barranquilla, Colombia (e-mail: jgonzalez@cuc.edu.co).

José Daniel Hernández-Vásquez Profesor de tiempo completo en la Universidad Antonio Nariño en la Facultad de Ingeniería Mecánica, Electrónica y Biomédica (FIMEB). Pertenece al grupo de investigación GIFOURIER Puerto Colombia, Colombia (e-mail: johernandez79@uan.edu.co). 
processos. A metodologia adotada, baseada na programação de algoritmos computacionais em linguagem C-Sharp (C\#), possibilitou: (i) realizar uma análise complexa de incerteza (GUM); (ii) estimar a curva de calibração ajustada a partir da teoria consagrada dos mínimos quadrados comuns e (iii) validar a partir de dados experimentais reais. Os resultados consolidados permitiram concluir que o software desenvolvido está alinhado aos requisitos da ISO / IEC 17025: 2017, além de ser uma ferramenta eficaz para aumentar a confiabilidade metrológica dos processos industriais, contribuindo para o avanço do conhecimento em metrologia.

Palabras claves - certificado de calibração, incerteza de medição, medição de temperatura, metrologia térmica, software em metrologia.

\section{INTRODUCCIÓN}

L OS instrumentos para la medición de temperatura (e.g.: termómetro de contacto, termopar, TLV, termómetro bimetálico) que son utilizados a nivel de laboratorio o industrial deben cumplir con especificaciones de calidad establecidas por una norma técnica o reglamento interno. Para conseguir este objetivo, la confiabilidad metrológica es evaluada a partir de un procedimiento de calibración. Los resultados obtenidos permiten conocer si los errores e incertidumbres reportadas cumplen con la tolerancia máxima definida para el proceso [1].

La metodología para la calibración de los termómetros de contacto es realizada a partir de (i) puntos fijos o (ii) comparación directa. Los puntos fijos son constantes naturales de los puntos de fusión, congelación, evaporación y puntos triples, organizados en la escala internacional de temperatura de 1990 (ITS-90). Debido a que son temperaturas constantes, éstos son tomados como referencia para la calibración del termómetro de trabajo [2]. Por otro lado, el método de comparación directa consiste en sumergir en un medio isotérmico, un termómetro patrón y el instrumento de trabajo para obtener las medidas de temperatura, así como los errores e incertidumbre asociada a la medición [3].

Los resultados de la calibración son consolidados y presentados en un certificado de calibración, que garantiza la confiabilidad de la trazabilidad metrológica [4]. El proceso de calibración ideal sugiere tomar infinitos puntos a lo largo del rango de medición del instrumento de trabajo. En la práctica, este procedimiento resulta imposible de llevar a cabo, así que un número de puntos finitos son considerados y distribuidos uniformemente, aumentado la incertidumbre de medición. En este sentido, en 2006 [5], así como en 2017 [6] proponen la generación de polinomios interpoladores o curvas de calibración que represente la naturaleza física de la calibración y matemáticamente permita estimar el error y la incertidumbre para aquellos puntos que no fueron considerados en la calibración, sin embargo, se encuentran dentro del rango de medición analizado.

En 2006, Chen [7] evaluó la incertidumbre asociada a una medición ajustada por medio de: (i) un polinomio de ajuste (grado uno y dos); (ii) función de potencia y (iii) ecuaciones de termómetros predefinidas en la literatura. Según [7] esta fuente de incertidumbre, llamada incertidumbre de ajuste, es calculada por el error medio cuadrático y debe ser evaluada para escoger la mejor curva de calibración o polinomio que mejor ajuste los datos experimentales.

La incertidumbre combinada de distintos tipos de termómetros (e.g.: Termómetro de resistencia de platina tipo PT-100 y Termopares) son calculadas a partir de su modelo de calibración y siguiendo el procedimiento sugerido por la Guía para la Expresión de la Incertidumbre de Medición (GUM 2008, del inglés, Guide to the Expression of Uncertainty in Measurement), conforme se muestra en otros trabajos [8], [9], [10].

El avance constante de la tecnología informática ha posibilitado un crecimiento en la ciencia de las mediciones. Así, en el campo de la metrología se han adoptado el uso de hojas de cálculo y softwares para el aseguramiento metrológico [11]. En estas herramientas se han incluido la automatización de las calibraciones, modelos matemáticos y sus resultados. Por ejemplo: la estimación de curvas de calibración por medio del método de los mínimos cuadrados ordinarios. Este método matemático es muy utilizado en metrología, ya que son muy flexibles al realizar la codificación de este [5] [12].

En la implementación de una Workstation de calibración de temperatura en un laboratorio de metrología en Eslovenia [13] fue diseñado un software, basado en la norma ISO/IEC 9126:1991, capaz de adquirir y procesar datos experimentales para, posteriormente, realizar el análisis matemático en base a normas y procedimientos previamente programados. Dentro de las principales ventajas, se constató que el software posibilitó la obtención de una curva de calibración, facilitó la generación de los certificados de calibración y permitió optimizar el tiempo de servicios, reduciendo errores de operación.

En 2001, el Laboratorio de Metrología y Calidad de la Universidad de Liubliana de Eslovenia desarrolló un sistema de calibración para termómetros de líquido en vidrio por medio de un módulo de adquisición de imagen con cámaras e iluminación. Este sistema permite la trasmisión de los datos adquiridos a un software de procesamiento de imagen, que traduce el nivel del líquido termométrico en temperatura, a través de una ecuación de interpolación [14].

Por otro lado, en el laboratorio metrológico del Instituto di Metrología G. Colonnetti (IMGC), todas las operaciones concernientes a la calibración de los patrones de termómetro de resistencia de platino (SPRT, por sus siglas en inglés: Standard Platinum Resistance Thermometers) en la escala de los puntos fijos ITS-90 son completamente automatizadas. El software está basado en el lenguaje de programación visual basic y desarrollado con ayuda de formularios y macros. Adicionalmente, este software se encuentra conectado con las aplicaciones de Microsoft Office para facilitar el tratamiento de datos en Excel y la posterior generación del certificado en Word. Además, cuenta con una consola virtual independiente para la adquisición de datos del procesamiento previo y posterior para, finalmente, entregar el producto final que es el certificado de calibración [15].

El Instituto tecnológico de Wuhan de China diseño un software para el análisis y tratamiento de los datos adquiridos en un procedimiento de calibración, a partir de la comparación directa entre el patrón de medición y un instrumento de tipo Termopar. Este software, además de permitir la adquisición de 
datos con baja señal de ruido, permite un control de la temperatura del baño por medio de un sistema PID (del inglés: Proportional, Integral and Derivative) y conexión al sistema LabVIEW. El algoritmo de programación permite la conversión de tensión eléctrica a unidades de temperatura. $\mathrm{Su}$ implementación ha incrementado la eficiencia del flujo de trabajo y la precisión de los resultados de calibración [16].

En 2011 (Ljungblad et al.) desarrollaron un software que almacena indicadores en su memoria interna durante un proceso de calibración. Posteriormente, es posible realizar un análisis estadístico de los datos, con alto grado de exactitud y precisión. Las mediciones tomadas por los indicadores son comparadas con las medidas de un termómetro digital patrón, lo que posibilita realizar cálculos para los errores, correcciones e incertidumbre. Al final del proceso, el software permite la generación de un certificado de calibración [17].

En base a la amplia revisión bibliográfica, fue constatado que el diseño de Softwares en Metrología se ha transformado en una alternativa tecnológica para el análisis de datos y aumento de la productividad de proceso. Además, han simplificado muchas tareas en el área de calibración de termómetros, no sólo han servido de complemento a los sistemas automatizados de calibración de termopares, también se han desarrollado software para el análisis de incertidumbre y generación de certificados de calibración para una amplia gama de termómetros.

En este contexto, el trabajo fue motivado por desafíos inherentes a la constante evolución de la metrología térmica a nivel industrial, cuyo principal objetivo es el diseño de un software para la generación de certificados de calibración de termómetros (termómetros digitales, líquido en vidrio, bimetálicos, termopares, PT-100) que cumpla con: (i) normas técnicas aplicadas para cada tecnología; (ii) procedimiento para el análisis y estimación de la incertidumbre de medición siguiendo los lineamiento de la GUM (2008); (iii) estándares y directrices definidas en la ISO/IEC 17025:2017.

\section{MODELO MATEMÁTICO PARA LA CURVA DE CALIBRACIÓN AJUSTADA}

Los termómetros de indicación directa como termómetros digitales, líquido en vidrio y bimetálicos, generalmente son modelados por una línea recta o una ecuación cuadrática [7]. Este trabajo propone evaluar polinomios grado 1, 2, e y 4. El grado de evaluación del polinomio está en función del número de datos experimentales que debe ser mayor al número de restricciones, i.e.: coeficientes de interpolación polinomial.

La curva de calibración para estos termómetros es generalizada por (1) en donde $t_{i}$ es la temperatura indicada por el termómetro calibrado y $t_{a d j}$ la temperatura ajustada, ésta representa la temperatura estimada del termómetro patrón con el que se realizó la calibración

$$
t_{a d j}=a_{0}+a_{1} t_{i}+a_{3} t_{i}^{2}+\cdots+a_{m} t_{i}^{m}
$$

El cálculo de los coeficientes se realiza aplicando el método de los mínimos cuadrados ordinarios matricialmente, para $\mathrm{N}$ pares de mediciones o puntos de calibración, en donde la variable independiente que, para el estudio propuesto, es la medición indicada por el termómetro calibrado $t_{i}$ y la variable dependiente $P_{i}$ la medición indicada por el termómetro patrón como se muestra en (2), en donde A es la matriz indicada en (3), a y b son vectores columna, y se expresan en (4) y (5) respectivamente.

$$
\begin{gathered}
a=A^{-1} b \\
A=\left(\begin{array}{ccccc}
N & \sum t_{i} & \sum t_{i}{ }^{2} & \cdots & \sum t_{i}{ }^{m} \\
\sum t_{i} & \sum t_{i}{ }^{2} & \sum t_{i}{ }^{3} & \cdots & \sum t_{i}{ }^{m+1} \\
\sum t_{i}{ }^{2} & \sum t_{i}{ }^{3} & \sum t_{i}{ }^{4} & \cdots & \sum t_{i}{ }^{m+2} \\
\vdots & \vdots & \vdots & \cdots & \vdots \\
\sum t_{i}{ }^{m} & \sum t_{i}{ }^{m+1} & \sum t_{i}{ }^{m+2} & \cdots & \sum t_{i}{ }^{m+m}
\end{array}\right) \\
a=\left(\begin{array}{c}
a_{0} \\
a_{1} \\
a_{2} \\
\vdots \\
a_{n}
\end{array}\right) \\
b=\left(\begin{array}{c}
\sum p_{i} t_{i} \\
\sum p_{i} t_{i}^{2} \\
\sum p_{i} t_{i}{ }^{3} \\
\vdots \\
\sum p_{i} t_{i}{ }^{n}
\end{array}\right)
\end{gathered}
$$

Los termopares son modelados por (6), el grado $\mathrm{N}$ del polinomio dependerá del rango de temperatura y puede variar entre 4 y 14. [10].

$E=\sum_{i=0}^{n} a_{i} t_{90}^{i}$

En las calibraciones por comparación de los termopares sin indicación resulta estratégicamente apropiado utilizar la función inversa de (6), una vez que, a partir de ésta, el usuario tiene la posibilidad de calcular la temperatura ajustada en función de la medición de la fuerza electromotriz $\mathrm{E}(\mathrm{mV})$ generada por el termopar como se observa en (7).

$t_{a d j}=\sum_{i=0}^{n} c_{i} \mathrm{E}^{i}$

La valoración de los coeficientes de la ecuación 4 también es realizada por medio del método de los mínimos cuadrados ordinarios, evaluando polinomios para grados entre 1 y 4.

Para la situación de termómetros de resistencia PT100 industriales sin indicación, éstos son modelados por la ecuación Callendar-Van Dusen [18], para temperatura por encima de $0^{\circ} \mathrm{C}$ expresada en (8).

$$
R(t)=R_{0}\left(1+A T+B T^{2}\right)
$$

Los coeficientes A y B son calculados por la matriz en (9), en donde $w\left(t_{i}\right)$ es la relación de la resistencia eléctrica de la termoresistencia a una temperatura $t_{i}$ dada, entre la resistencia eléctrica a $0^{\circ} \mathrm{C}$.

$$
\left[\begin{array}{l}
A \\
B
\end{array}\right]=\left[\begin{array}{ll}
\sum t_{i}^{2} & \sum t_{i}^{3} \\
\sum t_{i}^{3} & \sum t_{i}^{4}
\end{array}\right]^{-1}\left[\begin{array}{c}
\sum t_{i}\left(w\left(t_{i}\right)-1\right) \\
\sum t_{i}^{2}\left(w\left(t_{i}\right)-1\right)
\end{array}\right]
$$




\section{A. Criterio para selección de la curva de calibración}

Para evaluar los distintos ajustes obtenidos mediante el método de los mínimos cuadrados ordinarios, se debe calcular la dispersión de los datos en torno de la curva de regresión.

Para esta aplicación, el desvío medio cuadrático se torna un parámetro de decisión. En términos específicos, el desvío medio cuadrático resulta útil para comparar diferentes curvas de ajuste y, posteriormente, seleccionar aquella asociada a la menor incertidumbre de ajuste $u_{a}$, con el propósito de minimizar la incertidumbre combinada. La ecuación (10) presenta la expresión para determinar el desvío medio cuadrático o incertidumbre de ajuste:

$$
u_{\text {ajuste }}=\sqrt{\frac{\sum_{i=1}^{n}\left(y_{i}-y\left(x_{i}\right)\right)^{2}}{n-(m+1)}}
$$

Donde, $y_{i}$ es la variable dependiente obtenida experimentalmente (lectura del patrón), $y\left(x_{i}\right)$ es el valor de la temperatura ajustada, $n$ el número de puntos de calibración y $\mathrm{m}$ el grado del polinomio que se está evaluando. En esta ecuación, la sumatoria de los cuadrados de los residuos se dividen entre $n-(m+1)$, ya que $m+1$ coeficientes obtenidos de los datos se utilizaron para calcular los valores ajustados; así hemos perdido $m+1$ grados de libertad. Para el caso particular de los termómetros de resistencia del tipo PT-100, la incertidumbre de ajuste está asociada a la ecuación de Callendar-Van Dusen.

\section{ANÁLISIS DE INCERTIDUMBRE EN LA CALIBRACIÓN DE TERMÓMETROS}

La incertidumbre de medida es un parámetro asociado al resultado de una medición, que caracteriza la dispersión de los valores que podrían ser razonablemente atribuidos al mensurando, que en general comprende varios componentes, los cuales pueden ser evaluados y cuantificados mediante análisis estadísticos de series de observaciones de mediciones realizadas (El Método de Evaluación Tipo A) o evaluadas asumiendo distribuciones de probabilidad, basados en la experiencias adquiridas o investigaciones (Método de Evaluación Tipo B) (colocar REFERENCIA. GUM. 2008).

\section{A. Incertidumbre típica}

Es la incertidumbre asociada a la dispersión de las mediciones realizadas en torno a la media de estas. Estadísticamente se conoce como la desviación típica o estándar, indicada en (11).

$$
u_{q}=\sqrt{\frac{1}{n(n-1)} \sum_{j=1}^{n}\left(q_{j}-\bar{q}\right)^{2}}
$$

En donde $\left\{q_{1}, q_{2}, q_{3}, \ldots, q_{n}\right\}$ son las medidas tomadas durante el proceso de medición en un punto de calibración, $\bar{q}$ es la media de estas medidas y $\mathrm{n}$ es el número total de medidas realizadas en un punto de calibración.

\section{B. Incertidumbre de termómetro patrón}

Incertidumbre expandida $U_{P}$ obtenida del certificado de calibración con un factor de cobertura $\mathrm{k}$ indicado del termómetro patrón, tal como se indica en (12).

$$
u_{p}=U_{p} / k
$$

\section{Incertidumbre de lectura del termómetro a calibrar}

Resolución a del termómetro, puede ser digital o análoga y se le atribuye una distribución de probabilidad rectangular y triangular respectivamente.

$$
\begin{aligned}
& u_{R d}=a / \sqrt{12} \\
& u_{R a}=a / \sqrt{24}
\end{aligned}
$$

D. Incertidumbre por falta de uniformidad del baño de calibración

La uniformidad térmica de un medio de temperatura está caracterizada por la estabilidad $u_{\text {est }}$ y gradientes de temperatura $u_{\delta}$, encontradas en un estudio de caracterización de un medio isotérmico.

$$
u_{U N}=\sqrt{u_{e s t}^{2}+u_{\delta}^{2}}
$$

\section{E. Incertidumbre combinada}

En el caso de la calibración de un pt100, se debe hallar la relación de resistencia $\left(w\left(t_{i}\right)\right.$, es necesario medir dos magnitudes no correlacionadas $R\left(T_{90}\right)$ y $R(273,16 K)$. La incertidumbre combinada $u_{w}$ para $W\left(T_{90}\right)$ se calcula por medio de la ecuación.

$$
\left(\frac{u_{w}}{W}\right)^{2}=\left(\frac{u_{R}}{R}\right)^{2}+\left(\frac{u_{R_{0}}}{R_{0}}\right)^{2}
$$

En donde $u_{R}$ es la incertidumbre de medición de $R\left(T_{90}\right)$ y $u_{R_{0}}$ es la incertidumbre de medición de $R(273,16 K)$. Estas dos incertidumbres son combinaciones de la incertidumbre de la media y por la resolución del medidor de resistencia eléctrica utilizado.

Para hallar la incertidumbre debida a la resistencia $u_{T}$ en ${ }^{\circ} \mathrm{C}$ es necesario calcular el coeficiente de sensibilidad

$$
\begin{aligned}
& c_{T}=\frac{\partial T}{\partial W}=\frac{1}{A+2 B T} \\
& u_{T}=c_{T} u_{w}
\end{aligned}
$$

Cuando se trata de la calibración de un termopar es necesario medir su fuerza electromotriz generada por la diferencia de temperaturas entre su junta caliente y la junta de referencia a 0 ${ }^{\circ} \mathrm{C}$, para esto es necesario utilizar un voltímetro con una resolución en la escala de milivoltios. Generalmente, estos son calibrados y la incertidumbre asociada a su medición viene dada en sus certificados de calibración, esta incertidumbre es expandida $U_{E}$, por lo tanto, debe dividirse entre el factor de cobertura $k$ que indique el certificado de calibración.

Para que esta incertidumbre $u_{E}$ pueda ser expresada en grados Celsius, debe ser multiplicada por un coeficiente de sensibilidad $\frac{\partial t}{\partial E}$, que se obtiene al derivar el polinomio seleccionado para el termopar calibrado. 
En el caso de magnitudes de entrada no correlacionadas, la incertidumbre combinada $u_{c}(y)$ se calcula por la suma geométrica de las contribuciones particulares.

$$
u_{c}^{2}(y)=\sum_{i=1}^{N} u_{i}^{2}(y)
$$

Teniendo en cuenta este concepto y el coeficiente de sensibilidad, el cual relaciona el efecto que tiene la incertidumbre de una magnitud de entrada $x_{i}$ en el mensurando $y$. De esa manera determina que tan grande es la variabilidad del mensurando como resultado de la variabilidad (o incertidumbre) de esta magnitud de entrada, las incertidumbres para los termómetros de indicación directa (digitales, liquido en vidrio y bimetálicos), PT100 y termopares se indican en la Tabla 2.

TABLA I

INCERTIDUMBRES COMBINADAS PARA CADA TERMÓMETRO

\begin{tabular}{cc}
\hline \hline Termómetro & Incertidumbre combinada \\
\hline $\begin{array}{c}\text { Termómetros de } \\
\text { indicación } \\
\text { directa }\end{array}$ & $u_{c}{ }^{2}=u_{q}{ }^{2}+u_{p}{ }^{2}+u_{R}{ }^{2}+u_{U N}{ }^{2}+u_{\text {ajuste }}{ }^{2}$ \\
PT100 & $u_{c}{ }^{2}=\left(c_{T} u_{w}\right)^{2}+u_{p}{ }^{2}+u_{U N}{ }^{2}+u_{\text {ajuste }}{ }^{2}$ \\
Termopares & $u_{c}{ }^{2}=\left(\partial t / \partial E u_{E}\right)^{2}+u_{p}{ }^{2}+u_{U N}{ }^{2}+u_{\text {ajuste }}{ }^{2}$ \\
\hline \hline
\end{tabular}

\section{A. Factor de cobertura}

Frecuentemente, los valores del mensurando siguen una distribución normal. Sin embargo, el mejor estimado del mensurando, la media (obtenida por muestreos de $n$ mediciones repetidas) dividida entre su desviación estándar, se aproxima a una distribución llamada $t$-student, la cual refleja las limitaciones de la información disponible debidas al número finito de mediciones. Esta distribución coincide con la distribución normal en el límite cuando $n$ tiende a infinito, pero difiere considerablemente de ella cuando $n$ es pequeño.

$$
\frac{1}{v_{e f f}}=\sum_{i=1}^{N} \frac{\left(\frac{u_{i}(y)}{u_{c}(y)}\right)^{4}}{v_{i}}
$$

En la estimación de incertidumbres por el método tipo A, los grados de libertad de cada una de estas $v_{i}$ depende directamente del número de datos considerados y disminuye conforme el número de parámetros estimados a partir de los mismos datos. La repetibilidad de una medición, estimada por la desviación estándar experimental de $n$ lecturas tiene $n-1$ grados de libertad. Una regresión lineal de $M$ puntos mediante una ecuación de $m$ parámetros tiene $M-m$ grados de libertad. Si $u\left(x_{i}\right)$ se obtiene mediante evaluación Tipo $\mathrm{B}$, y puede considerarse como conocida con exactitud, lo que ocurre frecuentemente en la práctica, $v_{i} \rightarrow \infty$. En el caso de la distribución $t$-student el factor de cobertura estará en función de los grados efectivos de libertad y el nivel de confianza $p$.

En la calibración de termómetros es común utilizar un nivel de confianza de $95,45 \%$ y los grados de libertad efectivos $v_{\text {eff }}$ se estiman a partir de la ecuación de Welch-Satterthwaite en (20).

\section{Metodología}

El software TempSolutions 1.0, fue desarrollado en la plataforma de programación visual studio community 2015 en el lenguaje de programación $C \#$ integrando base de datos en SQL. El registro de derecho de autor fue realizado conforme a las políticas establecidas por el Ministerio del Interior de Colombia. Este software está conformado inicialmente por un formulario de control de usuario, una ventana principal con 8 opciones, de las cuales 5 dan el acceso a la calibración de termómetros bimetálicos, líquido en vidrio, PT-100, termopares y digitales. Las otras corresponden a los módulos de: administración de patrones y baños; controles de usuarios y configuraciones del certificado.

Al finalizar la calibración es generado un certificado (en formato PDF, Word o Excel) por medio de la función Report Viewer, conforme con los requisitos técnicos exigidos por la ISO/IEC 17025:2017.

Los pasos previos antes de seleccionar algún tipo de termómetro son los de registrar los patrones, baños y equipo auxiliar Medidor de resistencia eléctrica y voltímetro), en este formulario se ingresarán los datos básicos de cada equipo y las fuentes de incertidumbre de cada uno. Por su parte, en el formulario de usuario se ingresan los datos y la firma del metrólogo quien está autorizado a trabajar con el software. En el formulario de configuración se ingresan los datos básicos del laboratorio que utiliza el software y su logotipo. En la Fig. 1 se ilustra el esquema del software, indicando los datos de entrada, la transición interna de estos datos y la salida (Certificado de calibración). La Fig. 1 ilustra un esquema del software tempsolutions 1.0. 


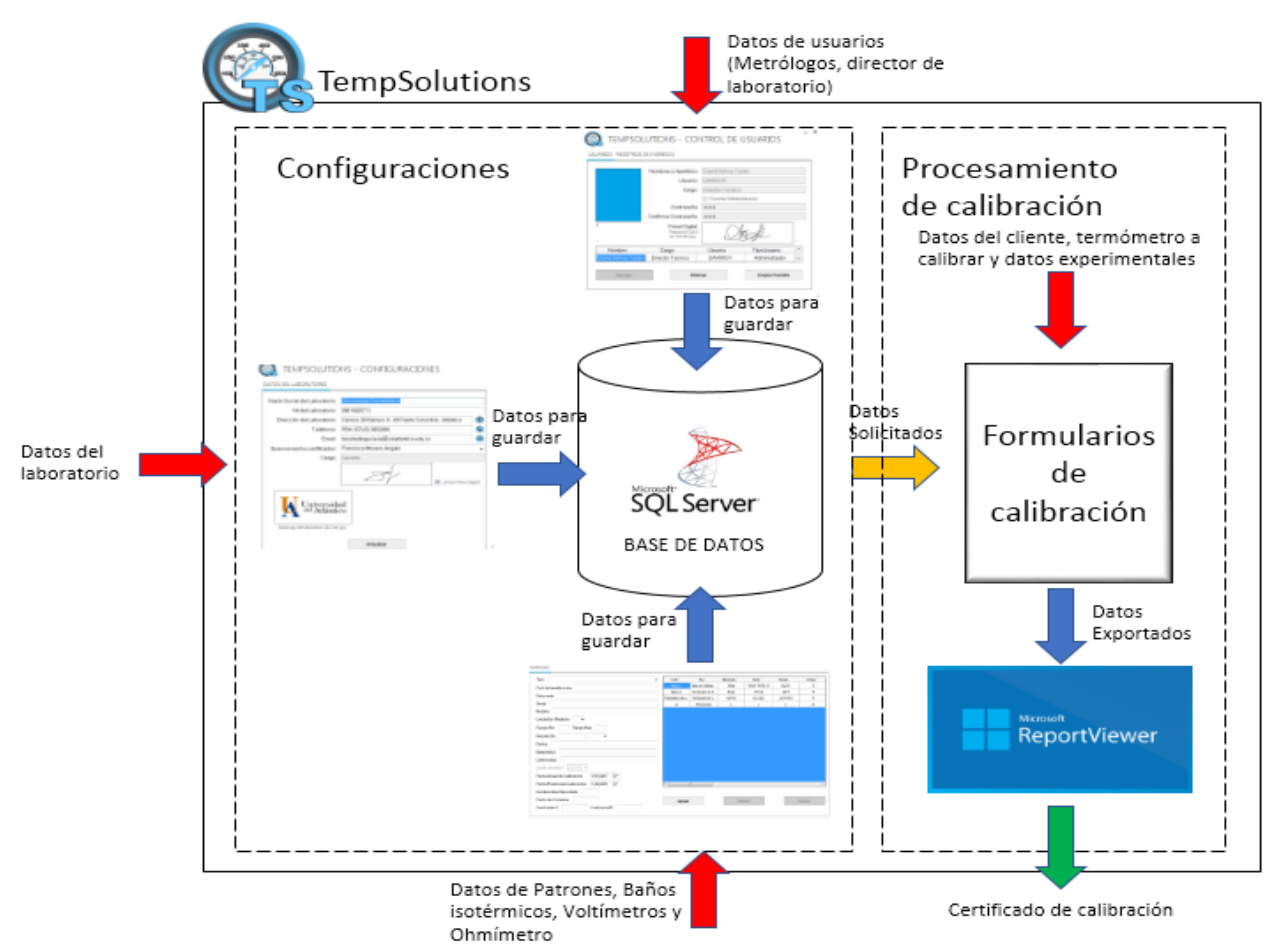

Fig. 1. Esquema del software tempsolutions 1.0

\section{A. Algoritmos}

En el paso de ingresar los datos experimentales obtenidos en la calibración, existe en la pestaña de ingreso de datos en el

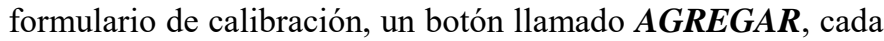
vez que es presionado se agregan las repeticiones en cada punto de calibración. En este software por parte del termómetro patrón son LP1, LP2 Y LP3 y las del termómetro a calibrar son

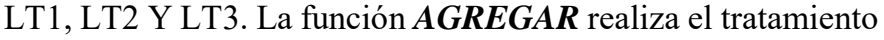
de datos para obtener los errores, correcciones, las incertidumbres, factor cobertura y el mejor polinomio de ajuste. El algoritmo se ilustra en los diagramas de bloques en la Fig.2 y Fig.3.

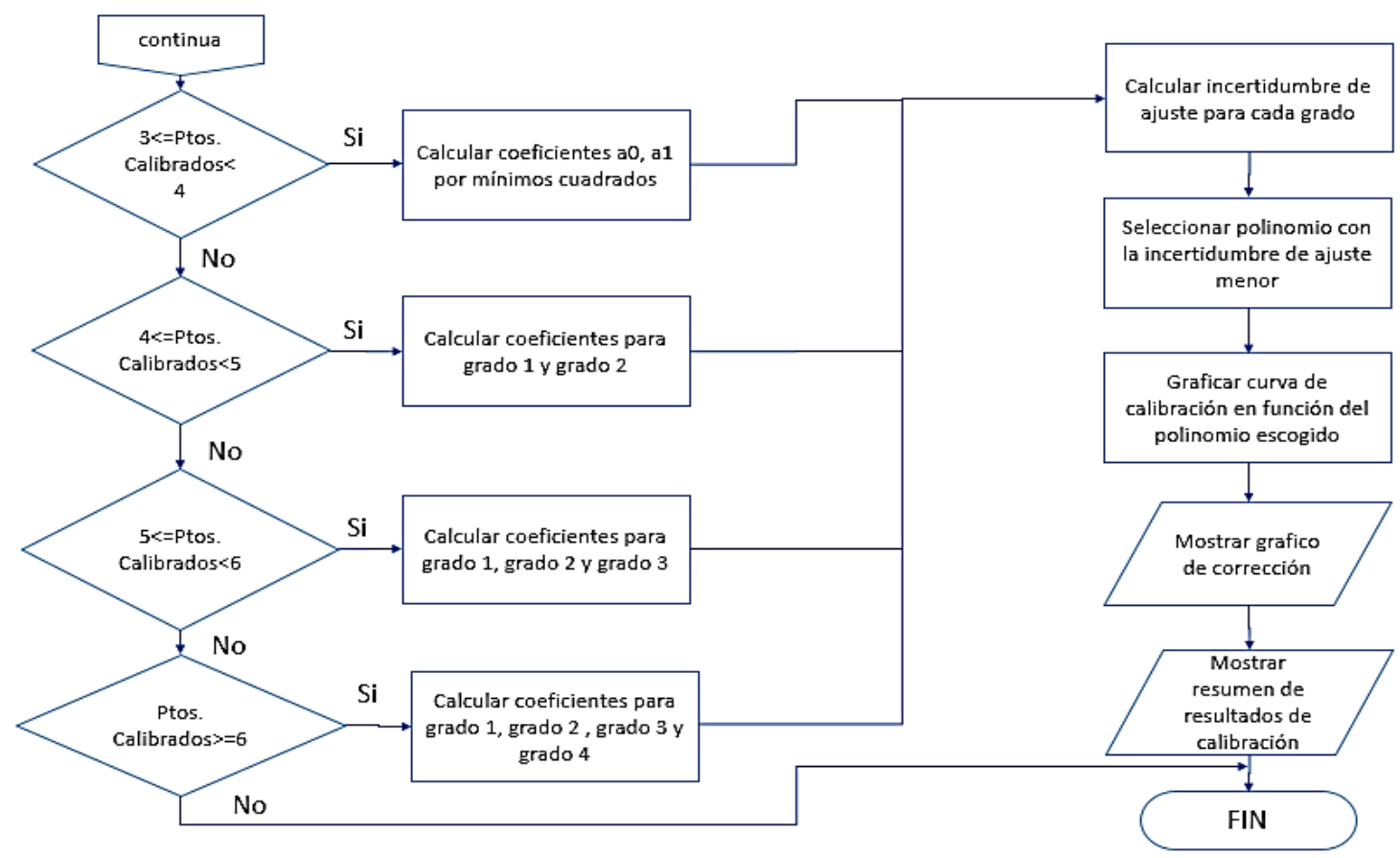

Fig. 2. Algoritmo de la función agregar 


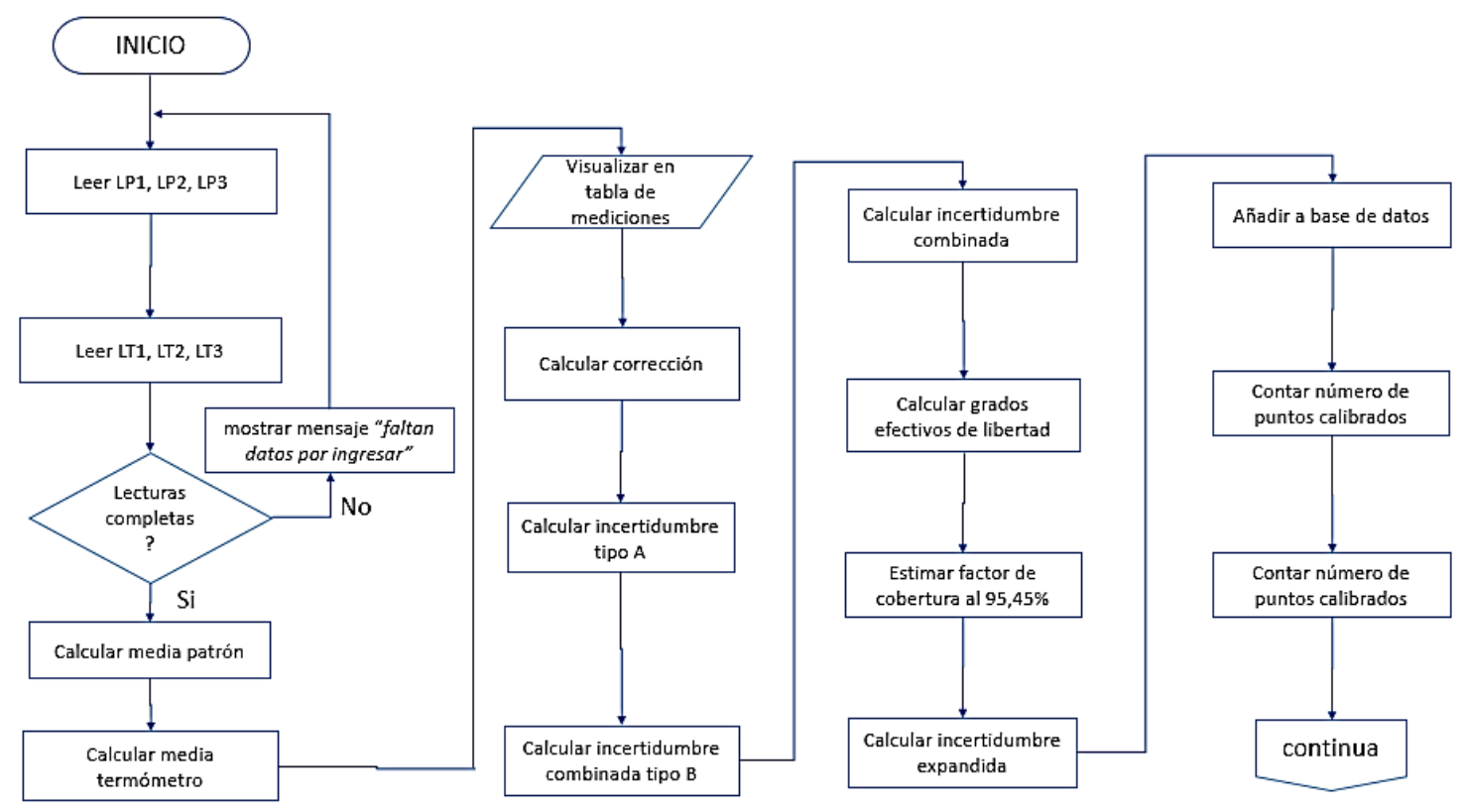

Fig. 3. Algoritmo de la función agregar (continuación)

\section{RESULTADOS Y DISCUSIÓN}

Por medio de los datos experimentales obtenidos de la calibración de un termómetro bimetálico se obtuvo que el mejor polinomio fue el de grado 3, evaluando las incertidumbres de ajuste de todos. Ver Tabla II, Tabla III y Tabla IV.

TABLA II

DATOS EXPERIMENTALES DE LA CALIBRACIÓN

\begin{tabular}{|c|c|c|c|}
\hline No. Mediciones & \multicolumn{2}{|c|}{$\begin{array}{l}\text { Indicación Del } \\
\text { Patrón }\left[{ }^{\circ} \mathrm{C}\right]\end{array}$} & ión Del \\
\hline 1 & \multicolumn{2}{|c|}{0,201} & 0 \\
\hline 2 & \multicolumn{2}{|c|}{19,900} &, 0 \\
\hline 3 & \multicolumn{2}{|c|}{39,899} &, 0 \\
\hline 4 & \multicolumn{2}{|c|}{59,799} &, 0 \\
\hline 5 & \multicolumn{2}{|c|}{80,100} &, 0 \\
\hline 6 & \multicolumn{2}{|c|}{100,102} & 0,0 \\
\hline \multicolumn{4}{|c|}{$\begin{array}{c}\text { TABLA III } \\
\text { TEMPERATURAS AJUSTADAS PARA CADA GRADO DE POLINOMIO } \\
\end{array}$} \\
\hline \multicolumn{4}{|c|}{ Temperatura Ajustada $\left[{ }^{\circ} \mathrm{C}\right]$ En Función De Cada Polinomio } \\
\hline Grado 1 & Grado 2 & Grado 3 & Grado 4 \\
\hline$-0,0002$ & 0,1619 & 0,2034 & 0,1927 \\
\hline 19,9998 & 19,9599 & 19,9154 & 19,9403 \\
\hline 39,9998 & 39,8379 & 39,8514 & 39,8063 \\
\hline 59,9998 & 59,7959 & 59,9154 & 59,8483 \\
\hline 79,9998 & 79,8339 & 80,0114 & 79,9703 \\
\hline 99,9998 & 99,9519 & 100,0434 & 99,9227 \\
\hline
\end{tabular}

TABLA IV INCERTIDUMBRES DE AJUSTE Y COEFICIENTES DEL MEJOR POLINOMIO

\begin{tabular}{clccc}
\hline \hline Grado del polinomio & 1 & 2 & 3 & 4 \\
\hline Incertidumbre de ajuste $\left[{ }^{\circ} \mathrm{C}\right]:$ & 0,17 & 0,18 & 0,10 & 0,25 \\
& $a_{0}$ & & 0,2034 \\
Mejor polinomio de ajuste (Grado 3): & $a_{1}$ & & 0,9784 \\
& $a_{2}$ & & 0,0004 \\
& $a_{3}$ & & $-0,0000017$ \\
\hline \hline
\end{tabular}

Para el cálculo de incertidumbre se tomaron los valores de las fuentes de incertidumbre mostrados en la Tabla V, la incertidumbre típica debida a la repetibilidad es 0 , ya que no se realizaron repeticiones

TABLA V

CÁlCULO DE INCERTIDUMBRE COMBINADA

\begin{tabular}{cccc}
\hline \hline Fuente de incertidumbre & $\begin{array}{c}\text { Valor } \\
{\left[{ }^{\circ} \mathrm{C}\right]}\end{array}$ & Incertidumbre $\left[{ }^{\circ} \mathrm{C}\right]$ & $u_{i}{ }^{2}$ \\
\hline$U_{P}$ & 0,0005 & 0,00025 & $6,25 \mathrm{E}-08$ \\
$a$ & 2,5 & 0,5 & 0,250 \\
estabilidad & 0,001 & 0,00057735 & $3,3333 \mathrm{E}-07$ \\
$\delta$ & 0,003 & 0,00173205 & 0,27 \\
$u_{\text {ajuste }}$ & 0,10 & 0,10 & 0,01367078 \\
\hline \multicolumn{4}{c}{ Resultados } \\
$u_{c}{ }^{2}(y)$ & \multicolumn{4}{c}{0,27409084} \\
\end{tabular}


Para el cálculo de la incertidumbre expandida, se realizó el cálculo del factor de cobertura, utilizando los grados efectivos de libertad, como se muestra en la Tabla VI.

TABLA VI

CÁLCULOS PARA LOS GRADOS EFECTIVOS DE LIBERTAD

\begin{tabular}{ccccc}
\hline Incertidumbre & $\boldsymbol{u}_{\boldsymbol{i}}(\boldsymbol{y})$ & $\boldsymbol{u}_{\boldsymbol{i}}(\boldsymbol{y})^{\mathbf{4}}$ & $\boldsymbol{v}_{\boldsymbol{i}}$ & $\frac{\boldsymbol{u}_{\boldsymbol{i}}(\boldsymbol{y})^{\mathbf{4}}}{\boldsymbol{v}_{\boldsymbol{i}}}$ \\
\hline$U_{P}$ & 0,00025 & $3,90625 \mathrm{E}-15$ & $\infty$ & 0 \\
$a$ & 0,5 & 0,06781684 & $\infty$ & 0 \\
estabilidad & 0,00173205 & $1,11111 \mathrm{E}-13$ & $\infty$ & 0 \\
$\delta$ & 0,00173205 & $9 \mathrm{E}-12$ & $\infty$ & 0 \\
$u_{\text {ajuste }}$ & 0,10 & 0,00018689 & 2 & $9,34451 \mathrm{E}-05$ \\
\hline
\end{tabular}

De lo anterior si reemplaza los valores en (20) y se despeja $v_{\text {eff }}$ se tiene un valor de 803.4 , dicho valor que tiende al infinito y con una probabilidad del $95,45 \%$ se tiene que el valor del factor de cobertura $k$ tiende a 2. Finalmente multiplicando el factor de cobertura por la incertidumbre combinada se calcula un valor de $1,04{ }^{\circ} \mathrm{C}$.

Como evidencia del resultado de esta calibración, se muestra el certificado de calibración generado finalmente por el software.

\section{CONCLUSIONES}

Este trabajo concibió el desarrollo de un software para la generación de certificados de calibración en el área de metrología térmica. Los resultados consolidados permitieron alcanzar plenamente y de forma exitosa este objetivo formulado, una vez que la normatividad vigente sobre los requisitos definidos en la ISO/IEC 17025:2005 fue plenamente atendida.

Modelos de calibración de los distintos termómetros para entregar el mejor polinomio interpolador que minimiza la incertidumbre calculada fueron definidos de forma detallada y fácilmente programados a través del lenguaje C-Sharp (C\#).

El software aquí diseñado puede ser utilizado en las calibraciones a nivel industrial, una vez que la metodología analítica para la estimación de incertidumbre sigue las directrices consagradas y definidas en la Guía para la expresión de la incertidumbre de medida (GUM).

Como contribución de este trabajo, se concluye que el software, denominado "TempSolutions 1.0" está apto para ser instalado en el sistema operativo de Windows, su interfaz de usuario resulta atractiva con una interacción basada en selección de menús y rellenado de formulario. Esto permite reducir el error humano y optimizar el tiempo para la realización de los cálculos de incertidumbre y la generación de los certificados de calibración en los laboratorios de metrología.
Climatic Chamber and Liquid Baths," Int. J. Thermophys., vol. 38, no. 100, 2017. DOI: 10.1007/s10765-017-2234-6

[4] R. Vendt, V. Vabson, T. Kübarsepp, and M. Noorma, "Traceability of temperature measurements in Estonia," Proc. Est. Acad. Sci., vol. 62, no. 2, pp. 116-121, 2013. DOI: 10.3176/proc.2013.2.05

[5] M. J. T. Milton, P. M. Harris, I. M. Smith, A. S. Brown, and B. A. Goody, "Implementation of a generalized least-squares method for determining calibration curves from data with general uncertainty structures,” Metrologia, vol. 43, no. 4, pp. S291-S298, 2006. DOI: $10.1088 / 0026-1394 / 43 / 4 / \mathrm{S} 17$

[6] D. White, "Propagation of Uncertainty and Comparison of Interpolation Schemes," Int. J. Thermophys., vol. 38, no. 39, 2017. DOI: $10.1007 / \mathrm{s} 10765-016-2174-6$

[7] C. Chen, "Evaluation of measurement uncertainty for thermometers with calibration equations," Accredit. Qual. Assur., vol. 11, no. 12, pp. 75-82, 2006. DOI: 10.1007/s00769-006-0109-9

[8] I. Lira, D. Camarano, J. Paredes, and F. Santiago, "Expression of the uncertainty of measurement in the calibration of thermometers. Part I: Standard platinum resistance thermometers," Metrologia, vol. 36, no. 2, pp. 107-111, 1999. DOI: 10.1088/0026-1394/36/2/5

[9] I. Yang, K. Soon, W. Joung, and Y. Kim, "Modelling the calibration of the industrial platinum-resistance thermometers according to GUM," Accredit. Qual. Assur., vol. 18, no. 4, pp. 359-362, 2013. DOI: $10.1007 / \mathrm{s} 00769-013-0962-2$

[10] I. Lira and P. R. Santos, "Expression of the uncertainty of measurement in the calibration of thermometers. Part II: Thermocouples," Metrologia, vol. 36, no. 5, pp. 415-419, 1999. DOI: $10.1088 / 0026-1394 / 36 / 5 / 3$

[11] D. Richter, "Software quality assurance in metrology," ISA Trans., vol. 33, no. 4, pp. 421-428, 1994. DOI: 10.1016/00190578(94)90025-6

[12] I. Lira, "Curve adjustment by the least-squares method," IOPscience, vol. 677, no. 6, pp. 677-681, 2000. DOI: $10.1088 / 0026-1394 / 37 / 6 / 5$

[13] [13] J. Bojkovski, J. Drnov`sek, I. Pu`snik, and T. Tasic, "Automation of a Precision Temperature Calibration Laboratory," IEEE Trans. Instrum. Meansurement, vol. 49, p. 6, 2000. DOI: $10.1109 / 19.850401$

[14] V. Batagelj, J. Bojkovski, J. Drnov`sek, and I. Pu`snik, "Automation of Reading Liquid-in-Glass Thermometers," IEEE Trans. Instrum. Meansurement, vol. 50, no. 6, pp. 1594-1598, 2001. DOI: $10.1109 / 19.982951$

[15] A. Merlone, P. Marcariono, P. P. M. Steur, and R. Dematteis, "From the fixed points calibration to the certificate: A completely automated temperature laboratory," in Advanced Mathematical and Computational Tools in Metrology VI, World Scientific Publishing Co, 2004, pp. 323-326. DOI: 10.1142/9789812702647_0034

[16] J. Chen, X. Hu, and L. Hu, "A New T hermocouple AutoCalibration System," in International Conference on Computer Science and Software Engineering, 2008, pp. 136-138. DOI: $10.1109 /$ CSSE.2008.856

[17] S. Ljungbland, L. Josefson, and H. M., "Method for Effective Calibration of Temperature Loggers with Automated Data," Int J Thermophys, vol. 32, no. 11, pp. 2495-2503, 2011. DOI: 10.1007/s10765-011-1042-7

[18] N. P. Molseeva, "Individual Calibration Of Resistance Thermometers For Measuring Temperature Difference," Meas. Tech., vol. 53, no. 9, pp. 1042-1046, 2010. DOI: 10.1007/s11018$010-9616-5$

\section{REFERENCIAS}

[1] L. Soler, F. Wieber, C. Allamel-Raffin, J. Gangloff, C. Dufour, and E. Trizio, "Calibration: A Conceptual Framework Applied to Scientific Practices Which Investigate Natural Phenomena by Means of Standardized Instruments," J. Gen. Philos. Sci., vol. 44, no. 2, pp. 263-317, 2013. DOI: 10.1007/s10838-013-9231-7

[2] G. Krapf, M. Schalles, and T. Fröhlich, "Estimation of fixed-point temperatures - A practical approach," Measurement, vol. 44, no. 2, pp. 385-390, 2011.DOI: 10.1016/j.measurement.2010.10.015

[3] V. Žužek and I. Pušnik, "Calibration of Air Thermometers in a

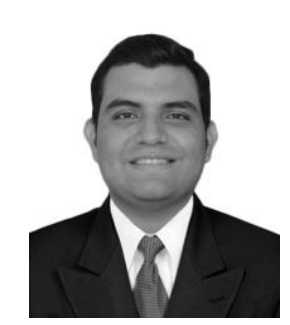

Francisco J. Moreno Angulo. Ingeniero Mecánico de la universidad del Atlántico, con experiencia en gestión de mantenimiento para maquinaria pesada e industrial. Ha trabajado en empresas del sector farmacéutico, minero y manufactura del aluminio. Ha participado en proyectos de construcción de plantas industriales en 
el área farmacéutica y posee conocimientos en la fundición del aluminio y su extrusión.

ORCID: https://orcid.org/0000-0002-1552-3520

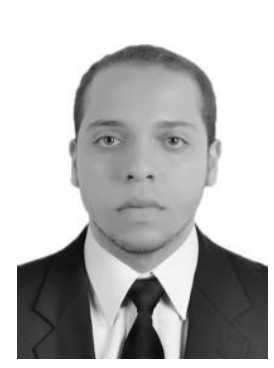

David Enrique Ochoa Tuirán. Ingeniero mecánico (Universidad del Atlántico, 2018). con experiencia en aseguramiento metrológico y Análisis de Sistemas de Medición (R\&R por variable y atributo, estabilidad y linealidad), estimación y expresión de la incertidumbre de medición. Experiencia en auditorías internas: ISO/IEC 17025:2005, ISO 9001:2015 y aseguramiento de calidad. Actualmente labora como ingeniero de metrología en Baterías Willard S.A. donde diseñó e implementó software de aseguramiento metrológico, desarrolla actividades de implementación de nuevos sistemas de medición con el fin de optimizar recursos y procesos. Realiza seguimiento a verificaciones y calibraciones en las variables de temperatura, masa, tensión, corriente, longitud, densidad y concentración.

ORCID: https://orcid.org/0000-0003-0337-2822

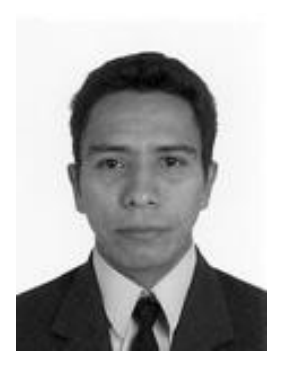

Cristian Antonio Pedraza Yepes. Nació en Barranquilla, Atlántico, Colombia en 1975. Recibió su título en Ingeniería Mecánica de la Universidad del Atlántico, Barranquilla, Colombia en 2001. Se graduó como Master en Ingeniería Mecánica de la Universidad del Norte, Barranquilla, Colombia en 2011 Actualmente trabaja como Profesor Asociado y miembro del Grupo de Investigación CONFORMAT del Programa de Ingeniería Mecánica de la Facultad de Ingeniería de la Universidad del Atlántico, Colombia. Las áreas de interés de investigación incluyen diseño mecánico, procesos de fabricación, caracterización de materiales y generación de energía alternativa.

ORCID: https://orcid.org/0000-0002-5951-7835

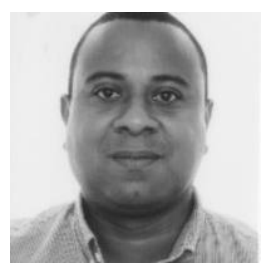

Jorge Enrique González Coneo. Ingeniero mecánico y magister en ingeniería mecánica de la Universidad del Norte. Docente tiempo completo de la Universidad de la Costa. Ha estado vinculado a los departamentos de Civil y Ambiental, Energía, y Ciencias Naturales y Exactas. Actualmente adelante estudios doctorales en Nanociencia y Tecnologías de Materiales. Ha trabajado en diferentes áreas: Mantenimiento, Materiales, Termofluidos e instrumentación.

ORCID: https://orcid.org/0000-0001-9391-8002

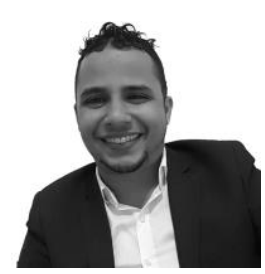

José Daniel Hernández Vásquez. Doctor en Ingeniería Mecánica, formado por la Pontificia Universidad Católica de Río de Janeiro (PUC-Río, 2018). Máster en Metrología (PUC-Rio, 2014). Ingeniero Mecánico (Universidad del Atlántico,
2011). Tiene experiencia docente a nivel de pregrado y postgrado, ministrando disciplinas en el Departamento de Ingeniería Eléctrica y el Programa de Posgrado en Metrología de la PUC-Río y la Facultad de Ingeniería de la Universidad del Atlántico. Tiene experiencia como investigador y consultor de la industria brasileña, actuando en proyectos financiados por el Organismo Brasileño Regulador del Sistema Eléctrico (ANEEL) y la Agencia Nacional de Petróleo, Gas Natural y Biocombustibles (ANP / PETROBRAS). Experiencia en la gestión técnica y administrativa de los laboratorios de metrología. Es reconocido por la Red Colombiana de Metrología (RCM, Sub-Red: Academia, Industria), como experto en metrología para el mercado colombiano. Actualmente, actúa como profesor de tiempo completo con dedicación exclusiva en la Facultad de Ingeniería Mecánica, Electrónica y Biomédica (FIMEB), Programa de Ingeniería Mecánica de la Universidad Antonio Nariño.

ORCID: https://orcid.org/0000-0002-0028-2912 